\title{
Reversible Surface Oxidation and Efficient Luminescence Quenching in Semiconductor Single Wall Carbon Nanotubes
}

\author{
Gordana Dukovic, Brian E. White, Zhiyong Zhou, Feng Wang, Steffen Jockusch, \\ Michael L. Steigerwald, Tony F. Heinz, Richard A. Friesner, Nicholas J. Turro, and \\ Louis E. Brus
}

\section{SUPPORTING INFORMATION}

\section{BAND-GAP ABSORPTION BLEACHING IN SWNTS DUE TO BAND-FILLING EFFECT}

The observed bleaching of band-gap absorption for nanotubes in an acidic environment can be understood by a band-filling effect: the free holes produced by the protonated surface oxide block the optical transitions that might generate these holes, and thus reduce the absorption strength. To estimate the required number of holes for a complete bleach of the band-gap absorption peak, we need to know the relevant electronic states responsible for this absorption. It has been predicted that exciton effects are strong in the prototype one-dimensional structure of SWNTs. ${ }^{1}$ A full calculation of the exciton bleaching arising from free-hole doping is demanding. We can, however, obtain a simple estimate by recalling that the exciton draws oscillator strength mostly from the transitions within the exciton binding energy from the band-edge and by using, accordingly, the approximation that the exciton transition will be bleached once the hole states corresponding to these transitions are filled. 
Within the free carrier picture, the density of states of 1-dimensional materials, such as SWNTs, exhibits a van Hove singularity at the band edge. The number of electronic transitions, and the corresponding number of hole states, up to an energy $D E$ from the band-edge is given by $N=D \frac{l}{\pi \hbar} \sqrt{m_{h} \Delta E}$, where 1 400nm is the length of our nanotubes and $\mathrm{D}=4$ is the degeneracy factor for $\mathrm{E}_{11}$ transition in semiconducting nanotubes. The hole effective mass $\left(\mathrm{m}_{\mathrm{h}}\right)$ for a carbon nanotube of $\sim 1 \mathrm{~nm}$ diameter is about $0.1 \mathrm{~m}_{0}$ in a tight-binding calculation. ${ }^{2}$ Assuming $D E \sim 100 \mathrm{meV}$, characteristic of the typical exciton binding energy, ${ }^{1}$ we estimate that about 250 holes are required to suppress the entire $E_{11}$ absorption peak. In this simple treatment we have not considered the effect of free-carrier screening on the electron-hole interactions, which may somewhat reduce the number of holes required to bleach the exciton absorption.

\section{REFERENCES:}

(1) Perebeinos, V.; Tersoff, J.; Avouris, P., arXiv:cond-mat/0402091.

(2) Saito, R.; Dresselhaus, G.; Dresselhaus, M. S. Physical properties of carbon nanotubes; Imperial College Press: London, 1998. 